\title{
On rotation of solar filaments
}

\author{
Khlystova A. I. ${ }^{1 \dagger}$ \\ ${ }^{1}$ Institute of Solar-Terrestrial Physics, P.O. Box 4026, Irkutsk, Russia \\ email: hlystova@iszf.irk.ru
}

\begin{abstract}
The study of the differential rotation law is an important issue in solar physics. Earlier studies of filaments found formulae for their differential rotation at stable existence stage. This paper relies on Big Bear Solar Observatory H-alpha filtergrams to estimate angular velocities of quiescent filaments at different stages of existence. Angular velocities are shown to cover a wide range of values. Likely causes of this velocity scatter are discussed.
\end{abstract}

\section{Method and the objects of investigation}

Full disk H-alpha filtergrams from Big Bear Solar Observatory were used to select 10 forming, 15 existing (for more than one half of the Sun rotation) and 12 decaying filaments for the years 2000 to 2005. Angular velocities in the filaments were traced for 105, 234 and 186 points, correspondingly. Thin filaments whose chief orientation was along the meridian were chosen. A filtergram set was selected for each filament covering its passage across the disk in the longitude range $\pm 40^{\circ}$ - one filtergram for each day.

The longitudinal position of a filament is found based on the profile minimum location in Fig. 1a (the part containing pixels corresponding to a chosen latitude near the filament). Ratio between the longitudinal difference in filament location in two images and the time difference was used to find the angular velocity of a filament at a given latitude (Fig. 1b). All the calculations employed the IDL programming language making use of Cartesian to/from spherical coordinate converting software accounting for the Sun rotation axis inclination B0 for each time moment. Mean angular velocity of the Earth's around the Sun rotation during filament observation was later added to the resulting synodic rotation velocities, yielding sidereal velocities of filament rotation (Fig. 2).

The errors were estimated as root-mean-square deviations.

\section{Results}

The obtained angular velocities of filament rotation span a wide range of values (Figs. 2 and 3 ). This velocity scatter may be related to:

1) the presence of proper motions in the filament because the tracer location (profile minimum) inside the filament varies from image to image (Fig. 2).

2) different depth of the roots of filament magnetic structures in the convective zone. One can see in Fig. 3 that average rotation velocities differ for individual filaments. It was shown by Thompson et al. (1996) that differential rotation of internal layers in the convective zone, within the latitudes range $\pm 40^{\circ}$, is not the same along the Sun radius. A differential component is not always observable in the filament motion. One can see in Fig. 2 that it is not for all the filaments that angular velocity decrease with increasing latitude is observable (Fig. 2). It is well known that filaments are located above polarityinversion lines. The dynamics of large-scale magnetic field can change the line's shape and this may give the effect of increased rotation velocity with increasing latitude.

$\dagger$ Present address: Institute of Solar-Terrestrial Physics, Lermontova, 126, Irkutsk, Russia. 

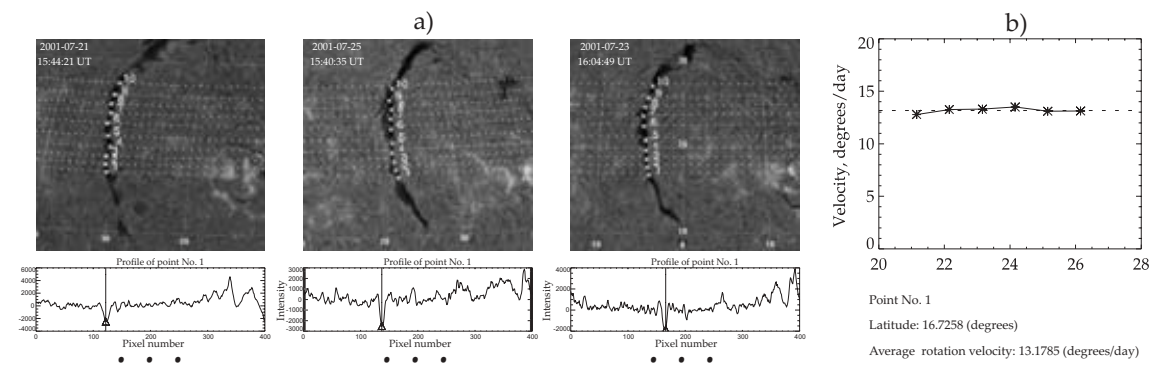

Figure 1. The filament transition through the disk from 2001.07.21 till 2001.07.26 in north hemisphere a) determination of the longitudinal position by the profile minimum b) velocity variation with time for the selected latitude.
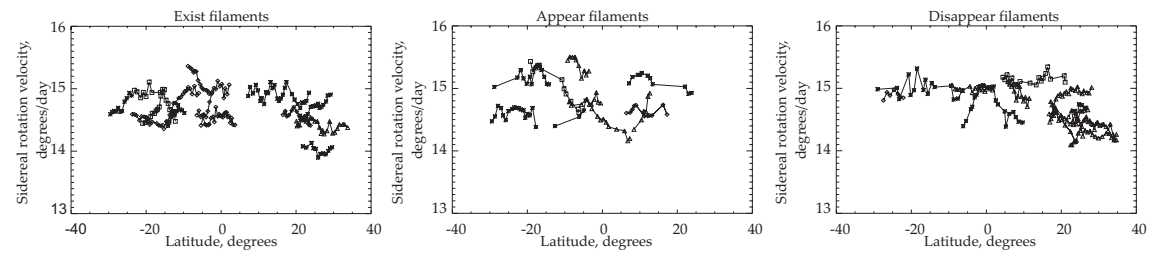

Figure 2. The latitude dependence of time-averaged angular velocities of the filaments. Points pertaining to the same filament are connected.

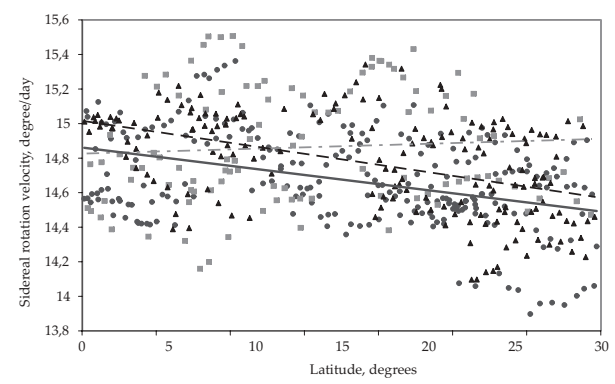

Figure 3. Linear approximation of the angular velocity for stable existing filaments (solid line), forming filaments (dotted line) and decaying filaments (dashed line).

The linear approximation of latitude dependencies of filament rotation velocities at various stages of existence shows that filaments rotate slower at stable-existence stage (Fig. 3). But the magnitude of this effect is at the level of the root-mean-square error which is $\pm 0.336^{\circ} /$ day, $\pm 0.273^{\circ} /$ day, and $\pm 0.287^{\circ} /$ day for the forming, stable-existence and decaying filament data correspondingly.

\section{Acknowledgements}

The author is grateful to Profs. Grigoryev V.M., Bashkirtsev B.S. and Mashnich G.P. for their help in the preparation of this work and useful discussions. This work was financially supported by Presidium of Russian Academy of Sciences Program No. 30 and RFBR grant 05-02-39017.

\section{Reference}

Thompson, M.J. et al. 1996, Science 272, 1300. 